\title{
TWO NEW MODELS FOR THE TWO-PERSON RED-AND-BLACK GAME
}

\author{
MAY-RU CHEN, ${ }^{*}$ National Sun Yat-sen University \\ SHOOU-REN HSIAU, ${ }^{* *}$ National Changhua University of Education
}

\begin{abstract}
In a two-person red-and-black game, each player holds an integral amount of chips. At each stage of the game, each player can bet any integral amount in his possession, winning the chips of his opponent with a probability which is a function of the ratio of his bet to the sum of both players' bets and is called a win probability function. Both players seek to maximize the probability of winning the entire fortune of his opponent. In this paper we propose two new models. In the first model, at each stage, there is a positive probability that two players exchange their bets. In the second model, the win probability functions are stage dependent. In both models, we obtain suitable conditions on the win probability functions such that it is a Nash equilibrium for the subfair player to play boldly and for the superfair player to play timidly.
\end{abstract}

Keywords: Two-person red-and-black game; win probability function; bold strategy; timid strategy; Nash equilibrium; convex function

2000 Mathematics Subject Classification: Primary 91A15

Secondary 91A05; $91 \mathrm{~A} 06$

\section{Introduction}

The red-and-black gambling problem, which has taken its name from the game of roulette, has interested probabilists for quite some time. In a discrete version of the red-and-black game, a player beginning with a positive integral fortune of $x$ units can stake any positive integer amount $a \leq x$. His fortune becomes $x+a$ if he wins with a fixed probability $w(0<w<1)$ and $x-a$ if he loses with probability $1-w$. The player seeks to maximize the probability of reaching a prespecified goal $M$ by gambling repeatedly with suitably chosen stakes. Dubins and Savage [3, pp. 87-89] showed that in the subfair case (i.e. $w \leq \frac{1}{2}$ ) an optimal strategy is bold play, which corresponds to always betting the entire fortune or just enough to reach the goal, whichever is smaller. This seems intuitively reasonable in that a shorter game seems to give a better chance to the subfair player since he will surely lose in the long run. In the superfair case (i.e. $w \geq \frac{1}{2}$ ), Ross [7] proved that it is optimal for the player to bet timidly, that is, always to stake 1 unit of his current fortune at each stage. Intuitively, if the player is superfair, it is better for him to prolong the game.

There have been some generalizations of the red-and-black game in several ways. A twoperson red-and-black game was introduced by Secchi [8]. Then Pontiggia [5] proposed two different formulations of two-person red-and-black models, in which two players hold a positive

Received 20 July 2009; revision received 24 November 2009.

* Postal address: Department of Applied Mathematics, National Sun Yat-sen University, 70 Lien-hai Road, Kaohsiung 804, Taiwan, R.O.C. Email address: mayru@faculty.nsysu.edu.tw

** Postal address: Department of Mathematics, National Changhua University of Education, No. 1, Jin-De Road, Changhua 500, Taiwan, R.O.C. Email address: srhsiau@cc.ncue.edu.tw 
integer fortune and they both aim to win the entire fortune of his opponent. One formulation is called a weighted two-person red-and-black game; the other one is called a proportional two-person red-and-black game. She showed that in each model, it is a Nash equilibrium for the subfair player to play boldly and for the superfair player to play timidly. Chen and Hsiau [2] extended Pontiggia's results to a two-person red-and-black game with bet-dependent win probability functions. They showed that if the subfair player's win probability function, $f$, is convex and satisfies $f(0)=0, f(s) \leq s$, and $f(s) f(t) \leq f(s t)$, then it is a Nash equilibrium for the subfair player to play boldly and for the superfair player to play timidly.

Pontiggia [5] also introduced an $N$-person model, called the proportional $N$-person redand-black game, in which a conjecture was proposed. Then Chen and Hsiau [2] gave a counterexample of the conjecture and Chen [1] showed that in the proportional three-person red-and-black game if the weights of players satisfy some suitable conditions then it is a Nash equilibrium for the subfair player to play boldly and for the superfair player to play timidly. Recently, Pontiggia [6] gave another general game, called an $N$-person nonconstant sum game. She showed that if the win probability function satisfies some suitable conditions then it is a Nash equilibrium for each player to play boldly.

In this paper we consider two new models of a two-person red-and-black game; one is a betexchangeable game and the other is a stage-dependent game. For simplicity, we will combine these two models into one version. Denote the two players by I and II. Let $M \geq 2$ be the total amount of chips in the system and let $S=\{0,1, \ldots, M\}$ be the state space of fortune of each player in the game. If player I has $x$ chips, let

$$
A_{\mathrm{I}}(x)= \begin{cases}\{1,2, \ldots, x\} & \text { if } x \in\{1, \ldots, M-1\}, \\ \{0\} & \text { if } x \in\{0, M\}\end{cases}
$$

be the action set for player I and let

$$
A_{\mathrm{II}}(x)= \begin{cases}\{1,2, \ldots, M-x\} & \text { if } x \in\{1, \ldots, M-1\}, \\ \{0\} & \text { if } x \in\{0, M\},\end{cases}
$$

be the action set for player II. Assume that each player chooses his action without any knowledge of the action chosen by the other. The goal of each player is to maximize his probability of taking all the chips, i.e. reaching $M$.

Let $\left\{f_{m}\right\}_{m \geq 1}$ be a sequence of nonzero functions from $[0,1]$ to $[0,1]$ with $f_{m}(0)=0$ and $f_{m}(s) \leq s$ for each $m \in \mathbb{N}$. Suppose that at stage $m$, player I has $x_{m}$ chips and bets $a_{m} \in A_{\mathrm{I}}\left(x_{m}\right)$ chips, while player II bets $b_{m} \in A_{\mathrm{II}}\left(x_{m}\right)$ chips. Let $0<w \leq 1$ and $\bar{w}=1-w$. The law of motion for player $\mathrm{I}$ is defined by

$$
x_{m+1}= \begin{cases}x_{m}+b_{m} & \text { with probability } f_{m}\left(\frac{a_{m}}{a_{m}+b_{m}}\right) w, \\ x_{m}-a_{m} & \text { with probability } 1-f_{m}\left(\frac{a_{m}}{a_{m}+b_{m}}\right), \\ x_{m}-a_{m}+b_{m} & \text { with probability } f_{m}\left(\frac{a_{m}}{a_{m}+b_{m}}\right) \bar{w},\end{cases}
$$

for $1 \leq x_{m} \leq M-1$ and by $x_{m+1}=x_{m}$ with probability 1 for $x_{m}=0$ or $x_{m}=M$. This means that once one of the players reaches $M$, the stage of neither player can change. Note that 
this model is more general than the model proposed by Chen and Hsiau [2] because the win probability functions are stage dependent and, after each bet, there is a positive probability that two players exchange their bets if $w<1$. Note that if $w=1$ and $f_{m}=f$ for all $m \in \mathbb{N}$, the above model is reduced to the model given in [2], which will be called a standard game with $f$.

Since $f_{m}(s) \leq s$, we see that

$$
\begin{aligned}
\mathrm{E}\left[x_{m+1} \mid x_{m}\right]= & \left(x_{m}+b_{m}\right) f_{m}\left(\frac{a_{m}}{a_{m}+b_{m}}\right) w+\left(x_{m}-a_{m}\right)\left[1-f_{m}\left(\frac{a_{m}}{a_{m}+b_{m}}\right)\right] \\
& +\left(x_{m}-a_{m}+b_{m}\right) f_{m}\left(\frac{a_{m}}{a_{m}+b_{m}}\right) \bar{w} \\
= & x_{m}+\left(a_{m} w+b_{m}\right) f_{m}\left(\frac{a_{m}}{a_{m}+b_{m}}\right)-a_{m} \\
= & x_{m}+\left(a_{m} w+b_{m}\right)\left[f_{m}\left(\frac{a_{m}}{a_{m}+b_{m}}\right)-\frac{a_{m}}{a_{m}+b_{m}}\right]-\frac{a_{m}^{2} \bar{w}}{a_{m}+b_{m}} \\
\leq & x_{m}
\end{aligned}
$$

for $1 \leq x_{m} \leq M-1$ and that $\mathrm{E}\left[x_{m+1} \mid x_{m}\right]=x_{m}$ if $x_{m}=0$ or $x_{m}=M$. Therefore, the process $\left\{x_{m}\right\}_{m \geq 1}$ of the fortune of player I is a supermartingale. This means that the game is subfair (or unfavorable) to player I and superfair (or favorable) to player II.

Hereafter, a timid strategy is that a player always stakes one chip at each stage of the game. A bold strategy is that a player always stakes his entire fortune at each stage of the game. The profile (bold, timid) will denote that player I always plays boldly and player II always plays timidly.

In the above general model, if $f_{m}=f$ for all $m \in \mathbb{N}$ and $0<w \leq 1$, the model is called a bet-exchangeable game with $(f, w)$. In Section 2 we will prove that if the profile (bold, timid) is a Nash equilibrium for a standard game with $f$, then (bold, timid) is also a Nash equilibrium for a bet-exchangeable game with $(f, w)$. In the above general model, if $w=1$, the model is called a stage-dependent game with $\left\{f_{m}\right\}_{m \geq 1}$. In Section 3 we will prove that if, for all $s \in[0,1], f_{1}(s) \geq f_{2}(s) \geq f_{3}(s) \geq \cdots$ and, for each $f_{m}$, the profile (bold, timid) is a Nash equilibrium for the standard game with $f_{m}$, then the profile (bold, timid) is a Nash equilibrium for the stage-dependent game with $\left\{f_{m}\right\}_{m \geq 1}$.

\section{Bet-exchangeable game}

In this section we consider a bet-exchangeable game with $(f, w)$. Suppose that at stage $m$, player I has $x_{m}$ chips and bets $a_{m} \in A_{\mathrm{I}}\left(x_{m}\right)$ chips, while player II bets $b_{m} \in A_{\mathrm{II}}\left(x_{m}\right)$ chips. The law of motion for player I is defined by

$$
x_{m+1}= \begin{cases}x_{m}+b_{m} & \text { with probability } f\left(\frac{a_{m}}{a_{m}+b_{m}}\right) w, \\ x_{m}-a_{m} & \text { with probability } 1-f\left(\frac{a_{m}}{a_{m}+b_{m}}\right), \\ x_{m}-a_{m}+b_{m} & \text { with probability } f\left(\frac{a_{m}}{a_{m}+b_{m}}\right) \bar{w},\end{cases}
$$

for $1 \leq x_{m} \leq M-1$ and by $x_{m+1}=x_{m}$ with probability 1 for $x_{m}=0$ or $x_{m}=M$. Note that if $w=1$ (that is, at each stage, the probability of players exchanging their bets is 0 ) then the 
bet-exchangeable game with $(f, w)$ is a standard game with $f$. For a bet-exchangeable game with $(f, w)$, the main result is the following, which extends the result in [2].

Theorem 2.1. If the profile (bold, timid) is a Nash equilibrium for a standard game with $f$ then (bold, timid) is also a Nash equilibrium for a bet-exchangeable game with $(f, w)$.

To prove Theorem 2.1, we need some results about a bet-exchangeable game with $(f, w)$. First, we place a certain condition on a bet-exchangeable game with $(f, w)$ to ensure that a bold strategy is optimal for player I when player II plays timidly.

Lemma 2.1. In a bet-exchangeable game with $(f, w)$, assume that player II adopts a timid strategy. If the win probability function $f$ satisfies

$$
f\left(\frac{a}{a+1}\right)+\left[1-f\left(\frac{a}{a+1}\right)\right] \prod_{i=0}^{a} f\left(\frac{x-i}{x-i+1}\right) \leq f\left(\frac{x}{x+1}\right)
$$

for all $x \in\{1, \ldots, M-1\}$ and $a \in A_{\mathrm{I}}(x)$, then a bold strategy is optimal for player $I$.

Proof. Assume that player II adopts a timid strategy. If player I uses a bold strategy, set

$$
Q(x)=\mathrm{P}(\text { player I reaches } M \text { with an initial fortune } x) .
$$

The corresponding law of motion at stage $m$ for player I having $x_{m}$ units and playing boldly is given by

$$
x_{m+1}= \begin{cases}x_{m}+1 & \text { with probability } f\left(\frac{x_{m}}{x_{m}+1}\right) w, \\ 0 & \text { with probability } 1-f\left(\frac{x_{m}}{x_{m}+1}\right), \\ 1 & \text { with probability } f\left(\frac{x_{m}}{x_{m}+1}\right) \bar{w},\end{cases}
$$

for $1 \leq x_{m} \leq M-1$, where $\bar{w}=1-w$, and by $x_{m+1}=x_{m}$ with probability 1 for $x_{m}=0$ or $x_{m}=M$. From this, it is not difficult to derive the recurrence relation

$$
Q(x)=f\left(r_{x}\right) w Q(x+1)+f\left(r_{x}\right) \bar{w} Q(1),
$$

where $r_{x}=x /(x+1)$ and $1 \leq x \leq M-1$. Note that $Q(0)=0$ and $Q(M)=1$.

A bold strategy is optimal for player I if and only if $Q(\cdot)$ is excessive (see Theorem 3.3.10 of [4]) or, equivalently, if the following inequality holds for every $1 \leq x \leq M-1$ and every $a \in A_{\mathrm{I}}$ :

$$
f\left(r_{a}\right) w Q(x+1)+\left(1-f\left(r_{a}\right)\right) Q(x-a)+f\left(r_{a}\right) \bar{w} Q(1) \leq Q(x) .
$$

Note that (2.3) holds if $a=x$, and so it remains to prove that (2.3) holds for $1 \leq a \leq x-1$.

Since $0<w \leq 1$, repeatedly using (2.2) yields

$$
\begin{aligned}
& Q(2)=\frac{1}{f\left(r_{1}\right) w}\left[Q(1)-f\left(r_{1}\right) \bar{w} Q(1)\right]=\left[1+\frac{1-f\left(r_{1}\right)}{f\left(r_{1}\right) w}\right] Q(1), \\
& Q(3)=\frac{1}{f\left(r_{2}\right) w}\left[Q(2)-f\left(r_{2}\right) \bar{w} Q(1)\right]=\left[1+\frac{1-f\left(r_{2}\right)}{f\left(r_{2}\right) w}+\frac{1-f\left(r_{1}\right)}{f\left(r_{1}\right) f\left(r_{2}\right) w^{2}}\right] Q(1),
\end{aligned}
$$


and so on. Then, by mathematical induction,

$$
Q(x)=\left[1+\sum_{k=1}^{x-1} \frac{1-f\left(r_{k}\right)}{w^{x-k} \prod_{i=k}^{x-1} f\left(r_{i}\right)}\right] Q(1) .
$$

Let $H(1)=1$, and, for $x \in\{2, \ldots, M-1\}$, let

$$
H(x)=1+\sum_{k=1}^{x-1} \frac{1-f\left(r_{k}\right)}{w^{x-k} \prod_{i=k}^{x-1} f\left(r_{i}\right)} .
$$

We obtain, for every $1 \leq x \leq M-1, Q(x)=H(x) Q(1)$ and

$$
H(x+1)=1+\frac{1-f\left(r_{x}\right)}{w f\left(r_{x}\right)}+\frac{1}{w f\left(r_{x}\right)} \sum_{k=1}^{x-1} \frac{1-f\left(r_{k}\right)}{w^{x-k} \prod_{i=k}^{x-1} f\left(r_{i}\right)}=\frac{H(x)}{w f\left(r_{x}\right)}-\frac{\bar{w}}{w} .
$$

Then, inequality (2.3) becomes

$$
f\left(r_{a}\right) w H(x+1) Q(1)+\left(1-f\left(r_{a}\right)\right) H(x-a) Q(1)+f\left(r_{a}\right) \bar{w} Q(1) \leq H(x) Q(1),
$$

which is equivalent to

$$
f\left(r_{a}\right) w H(x+1)+\left(1-f\left(r_{a}\right)\right) H(x-a)+f\left(r_{a}\right) \bar{w} \leq H(x)
$$

since $Q(1)>0$. Substituting (2.4) into (2.5) and then multiplying both sides by $f\left(r_{x}\right)$, where $f\left(r_{x}\right)>0$, we see that inequality $(2.5)$ is equivalent to

$$
f\left(r_{a}\right) H(x)+\left(1-f\left(r_{a}\right)\right) f\left(r_{x}\right) H(x-a) \leq f\left(r_{x}\right) H(x) .
$$

However, since $H(y) \geq 1 \geq f\left(r_{y}\right)$ for every $1 \leq y \leq M-1$ and $0<w \leq 1$, we see that

$$
H(y)=\frac{H(y)(1-\bar{w})}{w} \leq \frac{H(y)-f\left(r_{y}\right) \bar{w}}{w}=f\left(r_{y}\right)\left[\frac{H(y)}{w f\left(r_{y}\right)}-\frac{\bar{w}}{w}\right]=f\left(r_{y}\right) H(y+1) .
$$

The last equality holds as a result of (2.4). Repeatedly using (2.7) yields $H(x-a) \leq$ $\left[\prod_{i=1}^{a} f\left(r_{x-i}\right)\right] H(x)$. Thus, the left-hand side of inequality (2.6) satisfies

$$
f\left(r_{a}\right) H(x)+\left(1-f\left(r_{a}\right)\right) f\left(r_{x}\right) H(x-a) \leq H(x)\left[f\left(r_{a}\right)+\left(1-f\left(r_{a}\right)\right) \prod_{i=0}^{a} f\left(r_{x-i}\right)\right] .
$$

Because $f$ satisfies (2.1) and $H(x) \geq 1$, we have

$$
H(x)\left[f\left(r_{a}\right)+\left(1-f\left(r_{a}\right)\right) \prod_{i=0}^{a} f\left(r_{x-i}\right)\right] \leq H(x) f\left(r_{x}\right) .
$$

Then inequality (2.6) holds and so inequality (2.3) holds. Hence, the bold strategy is optimal for player I.

In the proof of Lemma 2.1, we see that if $w=1$ then, from (2.4), $H(y)=f\left(r_{y}\right) H(y+1)$ for all $1 \leq y \leq M-1$ and this implies that $H(x-a)=\left[\prod_{i=0}^{a} f\left(r_{x-i}\right)\right] H(x+1)$. Therefore, inequality (2.8) is actually an equality and so inequality (2.6) is equivalent to (2.1) since $H(x) \geq 1$. Thus, we have the following result. 
Theorem 2.2. In a standard game with $f$, assume that player II adopts a timid strategy. A bold strategy is optimal for player I if and only if $f$ satisfies (2.1) for all $x \in\{1, \ldots, M-1\}$ and $a \in A_{\mathrm{I}}(x)$.

Next, we place a certain condition on a bet-exchangeable game with $(f, w)$ to ensure that a timid strategy is optimal for player II when player I plays boldly.

Lemma 2.2. In a bet-exchangeable game with $(f, w)$, assume that player I plays a bold strategy. If the win probability function satisfies

$$
\prod_{i=0}^{b-1} f\left(\frac{x+i}{x+i+1}\right) \leq f\left(\frac{x}{x+b}\right)
$$

for all $x \in\{1, \ldots, M-1\}$ and $b \in A_{\mathrm{II}}(x)$, then a timid strategy is optimal for player II.

Proof. Assume that player I plays a bold strategy. If player II adopts a timid strategy, set

$$
T(x)=\mathrm{P}(\text { player II reaches } M \text { with an initial fortune } M-x) \text {. }
$$

Note that, after the $n$th bet, the probability that the game stops is greater than the probability of player II reaching $M$. However, after the $n$th bet, the probability of player II reaching $M$ is greater than $1-\left[f\left(\frac{1}{2}\right)\right]^{n}$. Note that the limit of $\left[f\left(\frac{1}{2}\right)\right]^{n}$ is 0 as $n$ goes to $\infty$. That is, the probability of player II reaching $M$ is going to 1 as $n \rightarrow \infty$ and so the game will eventually stop. Recalling $Q(x)$ defined in the proof of Lemma 2.1, we have $T(x)=1-Q(x)$. If at stage $m$ player II bids an amount $b$ then the corresponding law of motion for player I with $x_{m}$ chips is given by

$$
x_{m+1}= \begin{cases}x_{m}+b & \text { with probability } f\left(\frac{x_{m}}{x_{m}+b}\right) w, \\ 0 & \text { with probability } 1-f\left(\frac{x_{m}}{x_{m}+b}\right), \\ b & \text { with probability } f\left(\frac{x_{m}}{x_{m}+b}\right) \bar{w},\end{cases}
$$

for $1 \leq x_{m} \leq M-1$ and by $x_{m+1}=x_{m}$ with probability 1 if $x_{m}=0$ or $x_{m}=M$. As in the proof of Lemma 2.1, it suffices to prove that $T(\cdot)$ is excessive or, equivalently, that the following inequality holds:

$$
f\left(\frac{x}{x+b}\right) w T(x+b)+\left[1-f\left(\frac{x}{x+b}\right)\right] T(0)+f\left(\frac{x}{x+b}\right) \bar{w} T(b) \leq T(x) .
$$

Note that (2.10) holds if $b=1$, and then it remains to prove that (2.10) holds for $2 \leq b \leq M-x$.

Recall that $Q(x)=H(x) Q(1)$, where $H$ is defined in the proof of Lemma 2.1. Since $T(x)=1-Q(x)=1-H(x) Q(1), T(0)=1$, and $Q(1)>0$, inequality (2.10) becomes

$$
f\left(\frac{x}{x+b}\right) w H(x+b)+f\left(\frac{x}{x+b}\right) \bar{w} H(b) \geq H(x) .
$$


Substituting (2.4) into (2.11), we see that the right-hand side of (2.11) satisfies

$$
\begin{aligned}
& f\left(\frac{x}{x+b}\right) w H(x+b)+f\left(\frac{x}{x+b}\right) \bar{w} H(b) \\
& \quad=f\left(\frac{x}{x+b}\right)\left[\frac{H(x+b-1)}{f\left(r_{x+b-1}\right)}\right]+f\left(\frac{x}{x+b}\right) \bar{w}(H(b)-1) \\
& \quad \geq f\left(\frac{x}{x+b}\right)\left[\frac{H(x+b-1)}{f\left(r_{x+b-1}\right)}\right] .
\end{aligned}
$$

The last inequality holds since $f(x /(x+b)) \bar{w} \geq 0$ and $H(b) \geq 1$. Repeatedly using (2.7) yields $H(x+b-1) \geq H(x) /\left[\prod_{i=0}^{b-2} f\left(r_{x+i}\right)\right]$, and then

$$
f\left(\frac{x}{x+b}\right)\left[\frac{H(x+b-1)}{f\left(r_{x+b-1}\right)}\right] \geq f\left(\frac{x}{x+b}\right)\left[\frac{H(x)}{\prod_{i=0}^{b-1} f\left(r_{x+i}\right)}\right] .
$$

Since $f$ satisfies (2.9) and $H(x) \geq 1$, we have

$$
f\left(\frac{x}{x+b}\right)\left[\frac{H(x)}{\prod_{i=0}^{b-1} f\left(r_{x+i}\right)}\right] \geq H(x) .
$$

Hence, inequality (2.11) holds and so (2.10) holds. This completes the proof.

In the proof of Lemma 2.2, note that if $w=1$ then $\bar{w}=0$ and so inequality (2.12) is actually an equality. Moreover, recall that if $w=1$ then $H(y)=f\left(r_{y}\right) H(y+1)$ for all $1 \leq y \leq M-1$ and this implies that $H(x)=\left[\prod_{i=0}^{b-2} f\left(r_{x-i}\right)\right] H(x+b-1)$. Therefore, inequality $(2.13)$ is actually an equality and so inequality (2.11) is equivalent to (2.9) since $H(x) \geq 1$. Thus, we have the following result.

Theorem 2.3. In a standard game with $f$, assume that player I adopts a bold strategy. A timid strategy is optimal for player II if and only if $f$ satisfies (2.9) for all $x \in\{1, \ldots, M-1\}$ and $b \in A_{\mathrm{II}}(x)$.

In a bet-exchangeable game with $(f, w)$, suppose that $f$ satisfies (2.1) and (2.9). By Lemma 2.1, it follows that a bold strategy is optimal for player I if player II plays timidly; moreover, by Lemma 2.2, a timid strategy is optimal for player II if player I plays boldly. Therefore, it is a Nash equilibrium for player I to play boldly and for player II to play timidly. Hence, we have the following theorem.

Theorem 2.4. In a bet-exchangeable game with $(f, w)$, assume that the win probability function $f$ satisfies (2.1) and (2.9). Then the profile (bold, timid) is a Nash equilibrium for this game.

We now can prove Theorem 2.1 .

Proof of Theorem 2.1. If the profile (bold, timid) is a Nash equilibrium for a standard game with $f$ then, by Theorems 2.2 and 2.3, $f$ satisfies (2.1) and (2.9). Moreover, from Theorem 2.4, the profile (bold, timid) is also a Nash equilibrium for a bet-exchangeable game with $(f, w)$. This completes the proof.

From Theorem 2.3 of [2], it is known that the profile (bold, timid) is a Nash equilibrium for a standard game with $f$ if $f$ is convex and satisfies $f(s) f(t) \leq f(s t)$ for all $s, t \in(0,1)$. Then by Theorem 2.1 we have the following corollary. 
Corollary 2.1. In a bet-exchangeable game with $(f, w)$, if $f$ is convex and satisfies $f(s) f(t) \leq$ $f(s t)$ for all $s, t \in(0,1)$, then the profile (bold, timid) is a Nash equilibrium.

Remark 2.1. If we allow $w=0$ in a bet-exchangeable game, the case is very different. For example, if $w=0$ and $f(s)=s / 2$, then it is easy to verify that the game is subfair to player I and superfair to player II. Moreover, as in the proof of Lemma 2.1, we have $Q(x)=f\left(r_{x}\right) Q(1)$, where $r_{x}=x /(x+1)$. Therefore, for every $1 \leq x \leq M-1$ and every $1 \leq a \leq x-1$,

$$
\begin{aligned}
Q(x) & -\left[f\left(r_{a}\right) Q(1)+\left(1-f\left(r_{a}\right)\right) Q(x-a)\right] \\
& =\left[f\left(r_{x}\right)-f\left(r_{a}\right)-\left(1-f\left(r_{a}\right)\right) f\left(r_{x-a}\right)\right] Q(1) \\
& =-\left[\frac{a(x-a)(x+3)}{4(x+1)(a+1)(x-a+1)}\right] Q(1) \\
& <0 .
\end{aligned}
$$

This means that $Q(\cdot)$ is not excessive and, hence, a bold strategy is not optimal for player I while player II plays timidly. Similarly, it is also easy to verify that a timid strategy is not optimal for player II while player I plays boldly.

\section{Stage-dependent game}

In this section we consider a stage-dependent game with $\left\{f_{m}\right\}_{m \geq 1}$. Suppose that at stage $m$, player I has $x_{m}$ chips and bets $a_{m} \in A_{\mathrm{I}}\left(x_{m}\right)$ chips, while player II bets $b_{m} \in A_{\mathrm{II}}\left(x_{m}\right)$ chips. The law of motion for player I is defined by

$$
x_{m+1}= \begin{cases}x_{m}+b_{m} & \text { with probability } f_{m}\left(\frac{a_{m}}{a_{m}+b_{m}}\right), \\ x_{m}-a_{m} & \text { with probability } 1-f_{m}\left(\frac{a_{m}}{a_{m}+b_{m}}\right),\end{cases}
$$

for $1 \leq x_{m} \leq M-1$ and by $x_{m+1}=x_{m}$ with probability 1 for $x_{m}=0$ or $x_{m}=M$. For a stage-dependent game with $\left\{f_{m}\right\}_{m \geq 1}$, the main result is the following, which also extends the result in [2].

Theorem 3.1. If $f_{1}(s) \geq f_{2}(s) \geq f_{3}(s) \geq \cdots$ for all $s \in[0,1]$ and, for each $f_{m}$, (bold, timid) is a Nash equilibrium for a standard game with $f_{m}$, then (bold, timid) is a Nash equilibrium for a stage-dependent game with $\left\{f_{m}\right\}_{m \geq 1}$.

To prove Theorem 3.1, we need some results about a stage-dependent game with $\left\{f_{m}\right\}_{m \geq 1}$. First, we place a certain condition for a stage-dependent game with $\left\{f_{m}\right\}_{m \geq 1}$, which ensures that a bold strategy is optimal for player I while player II plays timidly.

Lemma 3.1. If $f_{1}(s) \geq f_{2}(s) \geq f_{3}(s) \geq \cdots$ for all $s \in[0,1]$ and each $f_{m}$ satisfies $(2.1)$, then in a stage-dependent game with $\left\{f_{m}\right\}_{m \geq 1}$, a bold strategy is optimal for player I while player II plays timidly.

Proof. Assume that player II plays a timid strategy. If player I uses a bold strategy, set

$$
Q_{m}(x)=\mathrm{P}(\text { player I reaches } M \text { with a fortune } x \text { at stage } m) \text {. }
$$


The corresponding law of motion at stage $m$ for player I having $x_{m}$ chips and playing boldly is given by

$$
x_{m+1}= \begin{cases}x_{m}+1 & \text { with probability } f_{m}\left(\frac{x_{m}}{x_{m}+1}\right), \\ 0 & \text { with probability } 1-f_{m}\left(\frac{x_{m}}{x_{m}+1}\right),\end{cases}
$$

for $1 \leq x_{m} \leq M-1$ and by $x_{m+1}=x_{m}$ with probability 1 for $x_{m}=0$ or $x_{m}=M$. From this, it is not difficult to derive the recurrence relation

$$
Q_{m}(x)=f_{m}\left(r_{x}\right) Q_{m+1}(x+1),
$$

where $1 \leq x \leq M-1$ and $r_{x}=x /(x+1)$. Note that $Q_{m}(0)=0$ and $Q_{m}(M)=1$ for all $m \in \mathbb{N}$.

In order to prove a bold strategy is optimal for player I while player II plays timidly, it suffices to show that the following inequality holds for every $x \in\{1, \ldots, M-1\}, a \in A_{\mathrm{I}}(x)$, and $m \in \mathbb{N}$ :

$$
f_{m}\left(r_{a}\right) Q_{m+1}(x+1)+\left[1-f_{m}\left(r_{a}\right)\right] Q_{m+1}(x-a) \leq Q_{m}(x) .
$$

Repeatedly using (3.1) yields

$$
Q_{m+1}(x+1)=f_{m+1}\left(r_{x+1}\right) Q_{m+2}(x+2)=\cdots=\prod_{i=1}^{M-x-1} f_{m+i}\left(r_{x+i}\right)
$$

and

$$
Q_{m+1}(x-a)=f_{m+1}\left(r_{x-a}\right) Q_{m+2}(x-a+1)=\cdots=\prod_{i=0}^{M-x+a-1} f_{m+i+1}\left(r_{x-a+i}\right) .
$$

Here we use $Q_{n}(M)=1$ for all $n \in \mathbb{N}$. Since $f_{1}(s) \geq f_{2}(s) \geq f_{3}(s) \geq \cdots$ for all $s \in[0,1]$, we have

$$
\begin{aligned}
\prod_{i=0}^{M-x+a-1} f_{m+i+1}\left(r_{x-a+i}\right) & =\left[\prod_{i=0}^{a} f_{m+i+1}\left(r_{x-a+i}\right)\right]\left[\prod_{j=1}^{M-x-1} f_{m+j+a+1}\left(r_{x+j}\right)\right] \\
& \leq\left[\prod_{i=0}^{a} f_{m}\left(r_{x-a+i}\right)\right]\left[\prod_{j=1}^{M-x-1} f_{m+j}\left(r_{x+j}\right)\right] \\
& =\left[\prod_{i=0}^{a} f_{m}\left(r_{x-i}\right)\right]\left[\prod_{j=1}^{M-x-1} f_{m+j}\left(r_{x+j}\right)\right] .
\end{aligned}
$$

The last identity holds since $\prod_{i=0}^{a} f_{m}\left(r_{x-a+i}\right)=\prod_{i=0}^{a} f_{m}\left(r_{x-i}\right)$. Moreover, since

$$
Q_{m+1}(x+1)=\prod_{i=1}^{M-x-1} f_{m+i}\left(r_{x+i}\right)
$$

we obtain

$$
Q_{m+1}(x-a) \leq\left[\prod_{i=0}^{a} f_{m}\left(r_{x-i}\right)\right] Q_{m+1}(x+1) .
$$


Substituting (3.3) and (3.4) into (3.2), we see that

$$
\begin{aligned}
f_{m}\left(r_{a}\right) & Q_{m+1}(x+1)+\left[1-f_{m}\left(r_{a}\right)\right] Q_{m+1}(x-a) \\
& \leq f_{m}\left(r_{a}\right) Q_{m+1}(x+1)+\left[1-f_{m}\left(r_{a}\right)\right]\left[\prod_{i=0}^{a} f_{m}\left(r_{x-i}\right)\right] Q_{m+1}(x+1) \\
& =\left\{f_{m}\left(r_{a}\right)+\left[1-f_{m}\left(r_{a}\right)\right] \prod_{i=0}^{a} f_{m}\left(r_{x-i}\right)\right\} Q_{m+1}(x+1) \\
& \leq f_{m}\left(r_{x}\right) Q_{m+1}(x+1) .
\end{aligned}
$$

The last inequality holds because $Q_{m+1}(x+1)>0$ and $f_{m}$ satisfies $(2.1)$, i.e.

$$
f_{m}\left(r_{a}\right)+\left[1-f_{m}\left(r_{a}\right)\right] \prod_{i=0}^{a} f_{m}\left(r_{x-i}\right) \leq f_{m}\left(r_{x}\right) .
$$

Since $f_{m}\left(r_{x}\right) Q_{m+1}(x+1)=Q_{m}(x),(3.2)$ holds. This completes the proof.

Next, we place a condition on the sequence of win probability functions such that a timid strategy is optimal for player II while player I plays boldly.

Lemma 3.2. If $f_{1}(s) \geq f_{2}(s) \geq f_{3}(s) \geq \cdots$ for all $s \in[0,1]$ and each $f_{m}$ satisfies (2.9), then in a stage-dependent game with $\left\{f_{m}\right\}_{m \geq 1}$, a timid strategy is optimal for player II while player I plays boldly.

Proof. Assume that player I plays a bold strategy. If player II adopts a timid strategy, let

$$
T_{m}(x)=\mathrm{P}(\text { player II reaches } M \text { with an initial fortune } M-x \text { at stage } m) .
$$

Recalling the functions $Q_{m}(x)$ defined in the proof of Lemma 3.1, we have $T_{m}(x)=1-Q_{m}(x)$. If at stage $m$ player II bids an amount $b$ then the corresponding law of motion for player I with $x_{m}$ chips is given by

$$
x_{m+1}= \begin{cases}x_{m}+b & \text { with probability } f_{m}\left(\frac{x_{m}}{x_{m}+b}\right) \\ 0 & \text { with probability } 1-f_{m}\left(\frac{x_{m}}{x_{m}+b}\right)\end{cases}
$$

for $1 \leq x_{m} \leq M-1$ and by $x_{m+1}=x_{m}$ with probability 1 for $x_{m}=0$ or $x_{m}=M$. As in the proof of Lemma 3.1, it suffices to prove that the following inequality holds for every $x \in\{1, \ldots, M-1\}, b \in A_{\mathrm{II}}(x)$, and $m \in \mathbb{N}$ :

$$
f_{m}\left(\frac{x}{x+b}\right) T_{m+1}(x+b)+\left[1-f_{m}\left(\frac{x}{x+b}\right)\right] T_{m+1}(0) \leq T_{m}(x) .
$$

Since $T_{m}(x)=1-Q_{m}(x)$ and $T_{m}(0)=1$ for all $m \in \mathbb{N}$, the above inequality becomes

$$
f_{m}\left(\frac{x}{x+b}\right) Q_{m+1}(x+b) \geq Q_{m}(x) .
$$


Recall that $r_{x}=x /(x+1)$. Repeatedly using (3.1) yields

$$
Q_{m}(x)=\prod_{i=0}^{M-x-1} f_{m+i}\left(r_{x+i}\right)
$$

and

$$
Q_{m+1}(x+b)=\prod_{i=b}^{M-x-1} f_{m+1+i-b}\left(r_{x+i}\right)=\prod_{i=b}^{M-x-1} f_{m+i-(b-1)}\left(r_{x+i}\right) .
$$

Here we use $Q_{n}(M)=1$ for all $n \in \mathbb{N}$. Since $b \geq 1$ and $f_{1}(s) \geq f_{2}(s) \geq f_{3}(s) \geq \cdots$ for all $s \in[0,1]$, we have

$$
\prod_{i=0}^{b-1} f_{m+i}\left(r_{x+i}\right) \leq \prod_{i=0}^{b-1} f_{m}\left(r_{x+i}\right)
$$

and

$$
\prod_{i=b}^{M-x-1} f_{m+i}\left(r_{x+i}\right) \leq \prod_{i=b}^{M-x-1} f_{m+i-(b-1)}\left(r_{x+i}\right)=Q_{m+1}(x+b) .
$$

Then

$$
\begin{aligned}
Q_{m}(x) & =\left[\prod_{i=0}^{b-1} f_{m+i}\left(r_{x+i}\right)\right]\left[\prod_{i=b}^{M-x-1} f_{m+i}\left(r_{x+i}\right)\right] \\
& \leq\left[\prod_{i=0}^{b-1} f_{m}\left(r_{x+i}\right)\right] Q_{m+1}(x+b) \\
& \leq f_{m}\left(\frac{x}{x+b}\right) Q_{m+1}(x+b) .
\end{aligned}
$$

The last inequality holds because $Q_{m+1}(x+b)>0$ and $f_{m}$ satisfies (2.9), i.e. $\prod_{i=0}^{b-1} f_{m}\left(r_{x+i}\right) \leq$ $f_{m}(x /(x+b))$. Hence, (3.5) holds. This completes the proof.

We can now prove Theorem 3.1.

Proof of Theorem 3.1. If the profile (bold, timid) is a Nash equilibrium for each standard game with $f_{m}$ then, by Theorems 2.2 and 2.3, each $f_{m}$ satisfies (2.1) and (2.9). Moreover, since $f_{1}(s) \geq f_{2}(s) \geq f_{3}(s) \geq \cdots$ for all $s \in[0,1]$, we find that in a stage-dependent game with $\left\{f_{m}\right\}_{m \geq 1}$, by Lemma 3.1, a bold strategy is optimal for player I while player II plays timidly and, by Lemma 3.2, a timid strategy is optimal for player II while player I plays boldly. Hence, the profile (bold, timid) is also a Nash equilibrium for a stage-dependent game with $\left\{f_{m}\right\}_{m \geq 1}$. This completes the proof.

From Theorem 2.3 of [2], it is known that the profile (bold, timid) is a Nash equilibrium for each standard game with $f_{m}$ if $f_{m}$ is convex and satisfies $f_{m}(s) f_{m}(t) \leq f_{m}(s t)$ for all $s, t \in[0,1]$. Thus, if $f_{1}(s) \geq f_{2}(s) \geq f_{3}(s) \geq \cdots$ for all $s \in[0,1]$ and each $f_{m}$ is convex and satisfies $f_{m}(s) f_{m}(t) \leq f_{m}(s t)$ for all $s, t \in[0,1]$, by Theorem 3.1, we have the following result.

Corollary 3.1. If $f_{1}(s) \geq f_{2}(s) \geq f_{3}(s) \geq \cdots$ for all $s \in[0,1]$ and each $f_{m}$ is convex and satisfies $f_{m}(s) f_{m}(t) \leq f_{m}(s t)$ for all $s, t \in[0,1]$, then the profile (bold, timid) is a Nash equilibrium for a stage-dependent game with $\left\{f_{m}\right\}_{m \geq 1}$. 


\section{Acknowledgements}

The first author gratefully acknowledges support from the National Science Council of Taiwan under grant NSC 97-2118-M-110-003-MY2 and from the National Center for Theoretical Sciences. The second author gratefully acknowledges support from the National Science Council of Taiwan under grant NSC 97-2118-M-018-002. They also wish to thank the anonymous referee for his/her helpful suggestions that improved the paper and made it more readable.

\section{References}

[1] Chen, M.-R. (2009). Proportional three-person red-and-black games. Prob. Eng. Inf. Sci. 23, 37-50.

[2] Chen, M.-R. and Hsiau, S.-R. (2006). Two-person red-and-black games with bet-dependent win probability functions. J. Appl. Prob. 43, 905-915.

[3] Dubins, L. E. And Savage, L. J. (1976). Inequalities for Stochastic Processes (How to Gamble if You Must). Corrected republication of the 1965 edition. Dover, New York.

[4] Maitra, A. P. and Sudderth, W. D. (1996). Discrete Gambling and Stochastic Games. Springer, New York.

[5] Pontiggia, L. (2005). Two-person red-and-black with bet-dependent win probability. Adv. Appl. Prob. 37, 75-89.

[6] Pontiggia, L. (2007). Nonconstant sum red-and-black games with bet-dependent win probability function. J. Appl. Prob. 44, 547-553.

[7] Ross, S. M. (1974). Dynamic programming and gambling models. Adv. Appl. Prob. 6, 598-606.

[8] Secchi, P. (1997). Two-person red-and-black stochastic games. J. Appl. Prob. 34, 107-126. 